\title{
The impact of MRI sequence on tumour staging and gross tumour volume delineation in squamous cell carcinoma of the anal canal
}

\author{
Davide Prezzi ${ }^{1,2,3}$ (D) Ramin Mandegaran ${ }^{2} \cdot$ Sofia Gourtsoyianni ${ }^{1,2}$. \\ Katarzyna Owczarczyk ${ }^{1,4}$ - Andrew Gaya ${ }^{4}$. Robert Glynne-Jones ${ }^{5} \cdot$ Vicky Goh $^{1,2,5}$
}

Received: 12 May 2017 /Revised: 30 August 2017 / Accepted: 22 September 2017 /Published online: 13 November 2017

(C) The Author(s) 2017. This article is an open access publication

\begin{abstract}
Objectives To compare maximum tumour diameter (MTD) and gross tumour volume (GTV) measurements between $T_{2^{-}}$ weighted $\left(\mathrm{T}_{2}-\mathrm{w}\right)$ and diffusion-weighted (DWI) MRI in squamous cell carcinoma of the anal canal (SCCA) and assess sequence impact on tumour (T) staging. Second, to evaluate interobserver agreement and reader delineation confidence. Methods The staging MRI scans of 45 SCCA patients (25 females) were assessed retrospectively by two independent radiologists ( 0 and 5 years' experience of anal cancer MRI). MTD and GTV were delineated on both $\mathrm{T}_{2}-\mathrm{w}$ and high-bvalue DWI images and compared between sequences; T staging was derived from MTD. Interobserver agreement was assessed and delineation confidence scored (1 to 5) by each observer.

Results GTV and MTD were significantly and systematically lower on DWI versus $\mathrm{T}_{2}$-W sequences by $14.80 \% / 9.98 \%$
\end{abstract}

Davide Prezzi

davide.prezzi@kcl.ac.uk

1 Division of Imaging Sciences \& Biomedical Engineering, King's College London, 4th Floor, Lambeth Wing, St. Thomas' Hospital, Westminster Bridge Road, London SE1 7EH, UK

2 Clinical Imaging, Guy's and St Thomas' NHS Foundation Trust, 1st Floor, Lambeth Wing, St. Thomas' Hospital, Westminster Bridge Road, London SE1 7EH, UK

3 Imaging 2, Level 1, Lambeth Wing, St Thomas' Hospital, Westminster Bridge Road, London SE1 7EH, UK

4 Clinical Oncology, Guy's and St Thomas' NHS Foundation Trust, Lambeth Wing, St. Thomas' Hospital, Westminster Bridge Road, London SE1 7EH, UK

5 Mount Vernon Cancer Centre, East and North Herts NHS Trust, Rickmansworth Rd, Northwood HA6 2RN, UK
(MTD) and 29.70\%/12.25\% (GTV) for each reader, respectively, causing $\mathrm{T}$ staging discordances in approximately a quarter of cases. Bland-Altman limits of agreement were narrower and intraclass correlation coefficients higher for DWI. Delineation confidence was greater on DWI: 40/42 cases were scored confidently (4 or 5 ) by each reader, respectively, versus $31 / 36$ cases based on $\mathrm{T}_{2}$-w images.

Conclusions Sequence selection affects SCCA measurements and $\mathrm{T}$ stage. DWI yields higher interobserver agreement and greater tumour delineation confidence.

Key Points

- MTD and GTV measurements are significantly lower on DWI than on $T_{2}-w$ MRI.

- Such differences cause T staging discordances in up to a quarter of cases.

- DWI results in higher agreement between inexperienced and experienced observers.

- DWI offers greater tumour delineation confidence to inexperienced readers.

Keywords Anus neoplasms · Magnetic resonance imaging · Neoplasm staging · Radiotherapy, image-guided · Diffusion magnetic resonance imaging
Abbreviations
DWI Diffusion-weighted imaging
GTV Gross tumour volume
IMRT Intensity-modulated radiotherapy
MTD Maximum tumour diameter
SCCA Squamous cell carcinoma of the anus
SIBR Simultaneous integrated boost radiotherapy
$\mathrm{T}_{2}$-W $\quad \mathrm{T}_{2}$ weighted 


\section{Introduction}

The incidence of squamous-cell carcinoma of the anus (SCCA), commonly referred to as anal cancer, has increased steadily over the past 4 decades in the Western world [1,2]. The standard-of-care treatment for non-metastatic SCCA is definitive chemoradiation (CRT) [3]: its aim is to eradicate the tumour while preserving anal sphincter function.

Magnetic resonance imaging (MRI) is recommended in Europe as the imaging modality of choice for loco-regional staging of SCCA [3] and has a growing role in radiation therapy planning [4]. High-resolution $\mathrm{T}_{2}$-weighted $\left(\mathrm{T}_{2}\right.$-w) sequences, obtained in the appropriate planes, provide detailed anatomical depiction of the anorectal region thanks to optimal soft-tissue contrast [5-8] and are in principle best suited for accurate target volume delineation.

Diffusion-weighted imaging (DWI) is now routinely included in body MRI protocols in most European oncological imaging centres: it has been shown to aid the diagnosis and response assessment of a variety of malignancies [9-13] and to allow the detection of small tumours in the pelvis [14]. Hypercellular tumours restrict water diffusion in the extracellular-extravascular space and typically stand out as bright lesions on a 'dark' background of suppressed signal on high $b$-value sequences, facilitating detection and delineation. Anal cancers typically appear restricted on DWI [15].

Maximum tumour diameter (MTD) is an important measurement in anal cancer, as it determines the T stage according to current TNM ( $7^{\text {th }}$ ed.) criteria [16] (Table 1). Gross tumour volume (GTV), defined as the gross primary anal tumour volume, forms the basis to calculate clinical and planning target volumes, which in turn determine radiotherapy dose distribution. Accurate GTV delineation is critical to the delivery of intensity-modulated radiotherapy (IMRT), which produces steep dose gradients and allows dose escalation to smaller high-risk target volumes (simultaneous integrated boost radiotherapy, SIBR) [17].

This study aimed to investigate the extent to which MRI measurements, specifically MTD and GTV, differ between anatomical $\mathrm{T}_{2}$-W and functional DWI sequences, as the implications for staging and treatment planning are clearly relevant

Table 1 SCCA primary tumour (T) staging criteria according to the AJCC Cancer Staging Manual, 7th edition [16]

$\begin{array}{ll}\text { TX } & \text { Primary tumour cannot be assessed } \\ \text { T0 } & \text { No evidence of primary tumour } \\ \text { Tis } & \text { Carcinoma in situ } \\ \text { T1 } & \text { Tumour } \leq 2 \mathrm{~cm} \text { in greatest dimension } \\ \text { T2 } & \text { Tumour }>2 \mathrm{~cm} \text { and } \leq 5 \mathrm{~cm} \text { in greatest dimension } \\ \text { T3 } & \text { Tumour }>5 \mathrm{~cm} \text { in greatest dimension } \\ \text { T4 } & \text { Tumour of any size invading adjacent organs }\end{array}$

to clinical practice. Second, it aimed to measure interobserver agreement for MTD and GTV as well as compare tumour detection confidence between observers with differing levels of interpretation experience.

\section{Materials and methods}

A review board waiver was granted for this retrospective analysis of anonymised imaging data acquired as part of normal clinical care. Fifty patients with biopsy-proven SCCA undergoing pelvic MRI for locoregional staging prior to definitive chemoradiation were identified from the picture archiving and communication system (PACS) of two tertiary-referral cancer centres, between July 2007 and June 2015. Cases were excluded if the tumour was incompletely imaged on either $\mathrm{T}_{2}$-w sequences or DWI $(n=3)$; the primary tumour was deemed undetectable on either sequence by secondary consensus reading $(n=2)$; the presence of MRI image artefact precluded accurate tumour measurements $(n=0)$.

\section{Imaging protocol}

Patients were scanned supine on one of three 1.5-T MRI scanners (Magnetom Avanto or Aera, Siemens Healthineers, Erlangen, Germany) using a pelvic phased array coil. The examination protocol included a $\mathrm{T}_{2}$-W sagittal turbo spin echo (TSE) sequence covering the pelvis (typical acquisition parameters: $\mathrm{TR} / \mathrm{TE}=4430 / 100 \mathrm{~ms}, \mathrm{NEX}=2, \mathrm{ST}=3 \mathrm{~mm}$, gap 0.3 $\mathrm{mm}$, FOV $250 \times 250 \mathrm{~mm}$, matrix $=307 \times 384)$, a $\mathrm{T}_{1}-\mathrm{w}$ axial TSE sequence for pelvic nodal detection $(\mathrm{TR} / \mathrm{TE}=552 / 11 \mathrm{~ms}$, $\mathrm{NEX}=1, \mathrm{ST}=5 \mathrm{~mm}$, gap $=1.5 \mathrm{~mm}, \mathrm{FOV}=300 \times 300 \mathrm{~mm}$, matrix $=240 \times 320$ ), a $\mathrm{T}_{2}$-W axial TSE sequence of the pelvis $(\mathrm{TR} / \mathrm{TE}=4590 / 101 \mathrm{~ms}, \mathrm{NEX}=1, \mathrm{ST}=5 \mathrm{~mm}$, gap $=1.5 \mathrm{~mm}$, FOV $=300 \times 300$, matrix $=307 \times 384)$ and high-resolution small-field-of-view $\mathrm{T}_{2}$-w TSE sequences perpendicular and parallel to the anal canal $(\mathrm{TR} / \mathrm{TE}=6530 / 104 \mathrm{~ms}, \mathrm{NEX}=2$, $\mathrm{ST}=3 \mathrm{~mm}$, gap $=0.3 \mathrm{~mm}, \mathrm{FOV}=200 \times 200$, matrix $=512 \times$ 512). DWI consisted of a single shot spin echo-echo planar imaging (SE-EPI) axial diffusion-weighted sequence (TR/TE $=5900 / 68 \mathrm{~ms}, \mathrm{NEX}=4, \mathrm{ST}=5 \mathrm{~mm}$, gap $=1.5 \mathrm{~mm}, \mathrm{FOV}=$ $300 \times 300$, matrix $=116 \times 154)$ encompassing the pelvis with three $b$-values in all cases $\left(0,100,800 \mathrm{~s} / \mathrm{mm}^{2}\right)$. Vendorgenerated apparent diffusion coefficient (ADC) maps were automatically created at the time of acquisition. Patients did not undergo any additional preparation prior to the examination.

\section{MTD and GTV measurements}

A third-year radiology resident (RM) with 1 year prior MRI experience but no previous experience in staging SCCA and a subspecialty gastrointestinal radiology fellow (DP) with 5 years' experience of staging gastrointestinal cancers evaluated 
the scans independently using all available sequences. Anonymised scans were downloaded from the local PACS onto a standalone workstation (iMac ${ }$, Apple Inc., CA, USA) and presented in randomised order in OsiriX v.7.5.1 (OsiriX Foundation, Geneva, Switzerland); readers were blinded to all clinical information. GTV delineation was performed separately on high-resolution axial-oblique $\mathrm{T}_{2}$-w and axial high-b-value $(b=800)$ DWI sequences, with a 1-week interval between the two reading sessions; DWI was read in conjunction with apparent diffusion coefficient (ADC) maps. Free-hand perilesional regions of interest (ROIs) were drawn on each slice with visible tumour and GTVs obtained by computing the ROI volumes. MTDs were obtained from sagittal $\mathrm{T}_{2}$-W sequences and sagittal reformats of axial high-b-value DWI, choosing the plane yielding the longest measurement on a case-by-case basis and using straight-line measurements (Fig. 1).

\section{GTV confidence score}

Each observer rated their confidence at contouring each tumour GTV on both $\mathrm{T}_{2}$-w and DWI sequences using a 5-point scale (1, no tumour boundaries identified with confidence; 2 , tumour boundaries identified with confidence on a minority of images $(<25 \%)$; 3 , tumour boundaries identified with confidence on approximately half of the images; 4 , tumour boundaries identified confidently on most images (>75\%); 5, tumour boundaries identified confidently on all images).

\section{Statistical analysis}

Statistical analysis was performed using IBM SPSS Statistics, version 23. Mean values between the two readers' MTD and GTV measurements were used in $\mathrm{T}_{2}$-W vs. DWI comparisons; measurements were compared using the independent samples t-test and correlated using Pearson's r. Interobserver agreement between the readers' MTD and GTV measurements was assessed using the 95\% Bland-Altman limits of agreement [18]. Intraclass correlation coefficients (two-way consistency model, absolute agreement type, average measures) were also calculated. A $P$ value $<0.05$ was taken to represent statistical significance for all analyses.

\section{Results}

The final cohort consisted of 45 patients, 25 females and 20 males, with a mean age of 62 years (standard deviation, 12.5;
Fig. 1 Anorectal SCCA. Highresolution $\mathrm{T}_{2}$-w axial-oblique image at the level of the anorectal junction (top left): lesion contouring is challenging considering the suboptimal contrast resolution between tumour and adjacent mucosa. Same-level high $b$-value axial DWI (top right): the hyperintense lesion can be clearly outlined against the suppressed signal of surrounding healthy tissue. $T_{2}-\mathrm{w}$ sagittal image used for MTD measurement (bottom left). Corresponding sagittal reformat of high $b$-value DWI, also used for MTD measurement (bottom right)
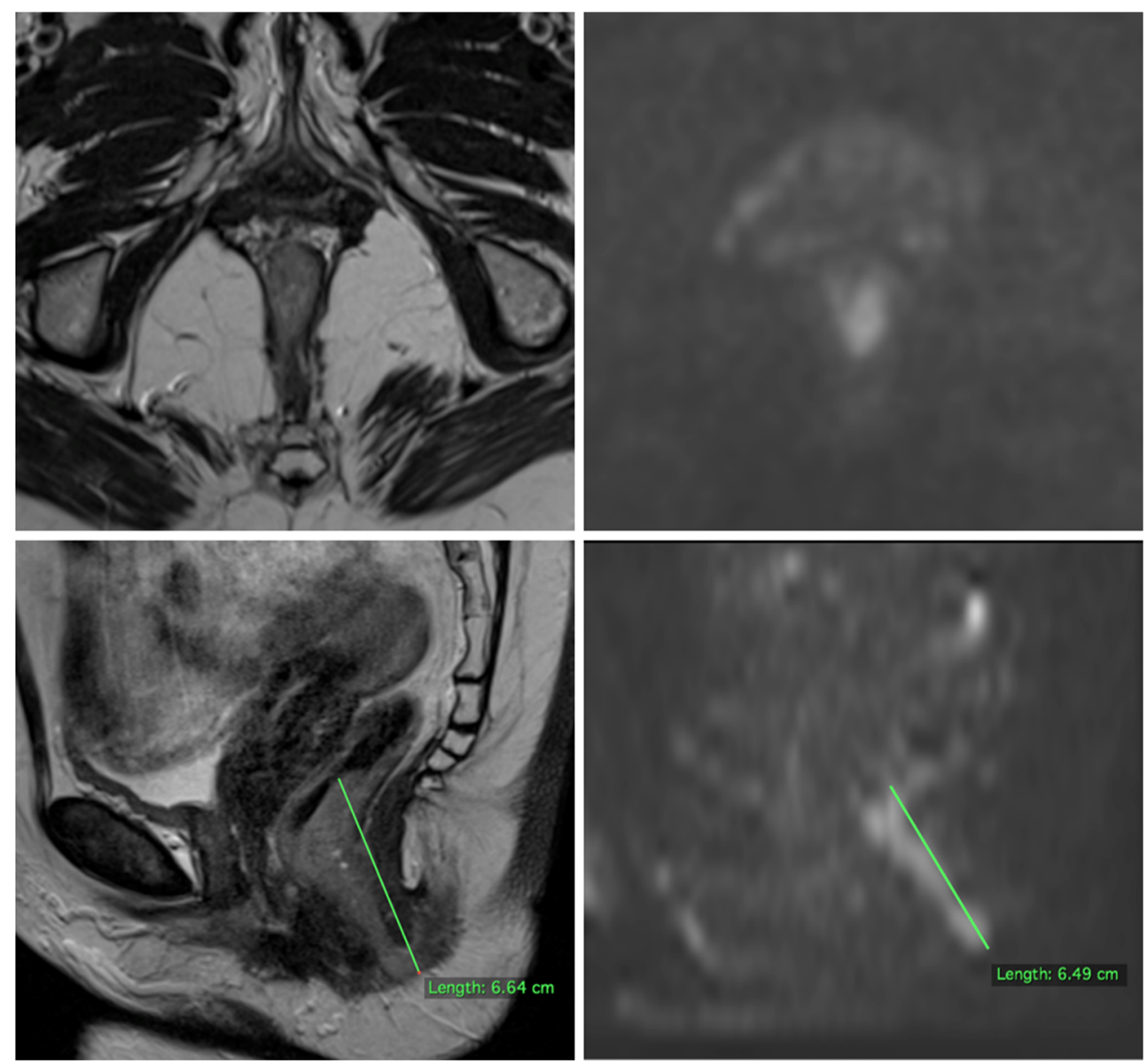
Table 2 Reader-specific MTD and GTV measurements (mean, standard deviation and range), paired samples t-test $P$ values and Pearson correlation test $\mathrm{r}$ and $P$ values

\begin{tabular}{|c|c|c|c|c|c|c|c|c|c|c|c|c|}
\hline & \multicolumn{6}{|c|}{ Observer 1 (inexperienced) } & \multicolumn{6}{|c|}{ Observer 2 (experienced) } \\
\hline & \multicolumn{3}{|c|}{ MTD (cm) } & \multicolumn{3}{|c|}{$\operatorname{GTV}\left(\mathrm{cm}^{3}\right)$} & \multicolumn{3}{|c|}{ MTD (cm) } & \multicolumn{3}{|c|}{ GTV $\left(\mathrm{cm}^{3}\right)$} \\
\hline & Mean & $\mathrm{SD}$ & Range & Mean & SD & Range & Mean & SD & Range & Mean & $\mathrm{SD}$ & Range \\
\hline $\mathrm{T}_{2}-\mathrm{w}$ & 5.88 & 2.14 & $1.36-10.93$ & 24.95 & 23.67 & $0.89-110.84$ & 5.41 & 2.07 & $1.46-9.58$ & 20.98 & 20.94 & $0.70-95.04$ \\
\hline DWI $b 800$ & 5.01 & 1.99 & $1.16-9.38$ & 17.54 & 18.30 & $0.16-79.99$ & 4.87 & 2.24 & $0.79-10.83$ & 18.41 & 20.10 & $0.13-95.15$ \\
\hline $\begin{array}{l}\text { Relative } \\
\text { change }\end{array}$ & $-14.80 \%$ & & & $-29.70 \%$ & & & $-9.98 \%$ & & & $-12.25 \%$ & & \\
\hline t-test $P$ & $<0.001$ & & & $<0.001$ & & & $<0.001$ & & & $<0.001$ & & \\
\hline Correlation $r$ & 0.875 & & & 0.949 & & & 0.906 & & & 0.987 & & \\
\hline Correlation $P$ & $<0.001$ & & & $<0.001$ & & & $<0.001$ & & & $<0.001$ & & \\
\hline
\end{tabular}

range, 37-84 years) and corresponding to $45 \mathrm{MRI}$ data sets analysed by each observer.

\section{MTD and GTV measurements}

Reader-specific tumour diameters and volumes measured on $\mathrm{T}_{2}$-W sequences and DWI are summarised in Table 2. GTV and MTD measurements were significantly different between $\mathrm{T}_{2}$-W and DWI for both observers (paired samples t-test $P$ values $<0.001$ ) (Table 2) and consistently lower on DWI (Fig. 2) by percentage values ranging between $9.98 \%$ and $29.70 \%$ (Table 2). As a consequence, MTD-based tumour (T) staging was discordant in 12 cases based on inexperienced observer measurements and in 10 cases based on experienced measurements (Fig. 3). As expected, inter-sequence measurements were strongly and significantly correlated, with $r$ values ranging between 0.875 and 0.987 .

\section{Interobserver agreement}

Agreement was marginally superior on DWI for MTD. Mean MTD difference (95\% limits of agreement) between the two readers was $-0.46(-2.89$ to +1.97$) \mathrm{cm}$ on $\mathrm{T}_{2}-\mathrm{W}$ and $-0.14(-$

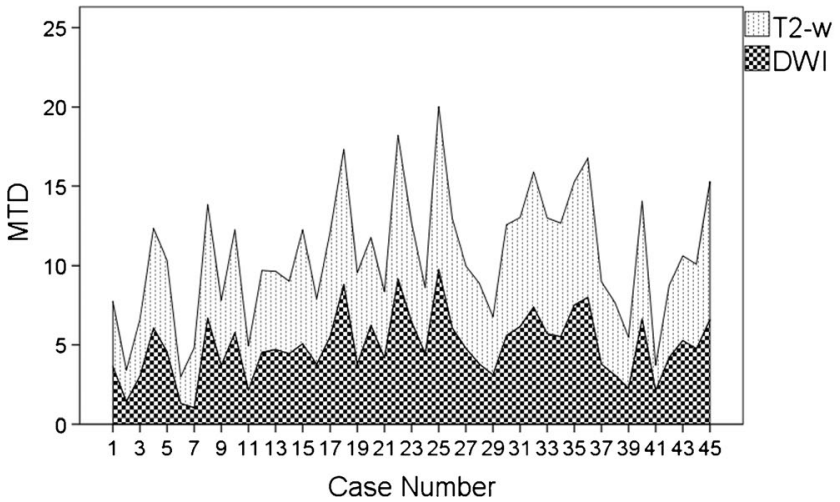

2.38 to +2.10$) \mathrm{cm}$ on DWI. Agreement was considerably superior on DWI for GTV. Mean GTV difference was -3.96 (17.91 to +9.97$) \mathrm{cm}^{3}$ on $\mathrm{T}_{2}$-W and $0.87(-6.75$ to +8.50$) \mathrm{cm}^{3}$ on DWI (Fig. 4). Intraclass correlation coefficients, reported in Table 3, were well above 0.8 (indicating excellent agreement) but higher for DWI.

\section{GTV confidence scoring}

Tumours were outlined with greater confidence on DWI than on $T_{2}-\mathrm{W}$ sequences by both readers. This gap in confidence was more substantial for the inexperienced reader: they assigned a low confidence score ( 1 to 3 ) to 14 cases on $T_{2}-\mathrm{W}$ versus 5 cases for the experienced reader on DWI and a high confidence score to 31 cases on $\mathrm{T}_{2}-\mathrm{W}$ versus 40 cases on DWI. Full confidence score results are reported in Figs. 4 and 5.

\section{Discussion}

We found that tumour volumes and maximum diameters measured on functional DWI were significantly lower than those measured on anatomical $\mathrm{T}_{2}-\mathrm{W}$ sequences.

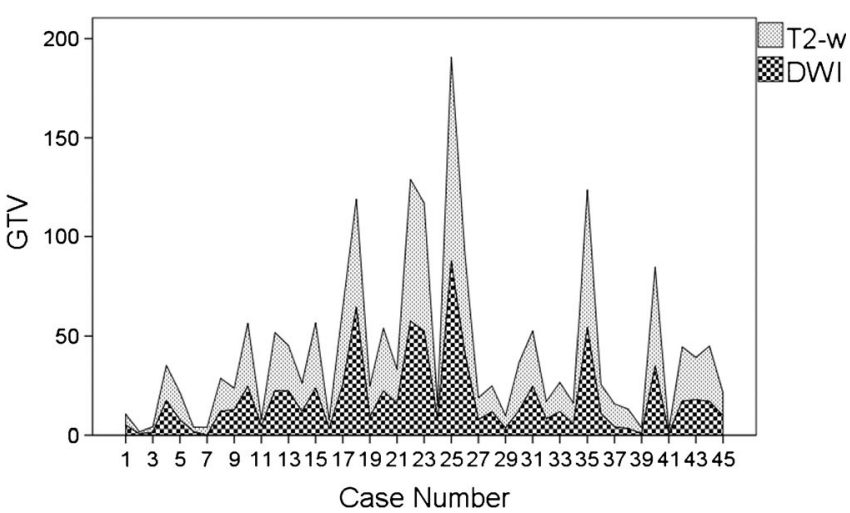

Fig. 2 Mean MTD $(\mathrm{cm})$ and GTV $\left(\mathrm{cm}^{3}\right)$ measurements on $T_{2}$-W versus DWI sequences, visualised case by case. Both measurements were systematically lower on DWI than on $\mathrm{T}_{2}$-W 

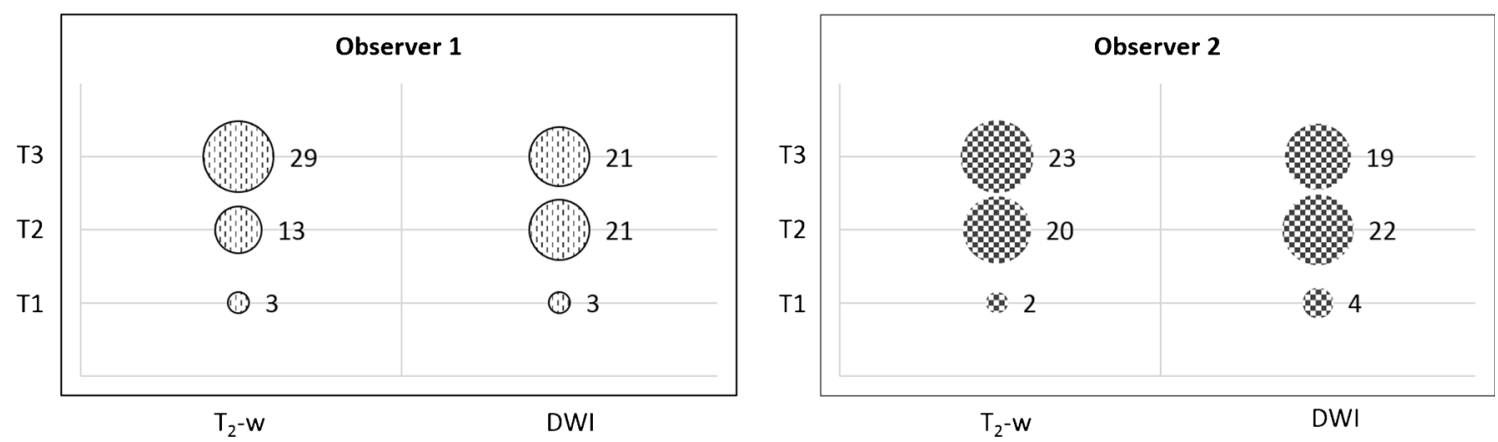

Fig. 3 Tumour (T) staging based on MTD measurements on T2-w versus DWI. Sequence selection affects T staging, particularly when the reader is inexperienced (Observer 1)

While no previous paper has investigated anal cancer, similar differences have been found in primary rectal cancer [19-21]: Curvo-Semedo et al. reported median pre-treatment tumour volumes of $18.0 \mathrm{~cm}^{3}$ on DWI versus $21.8 \mathrm{~cm}^{3}$ on $\mathrm{T}_{2^{-}}$

\section{T2 MTD}

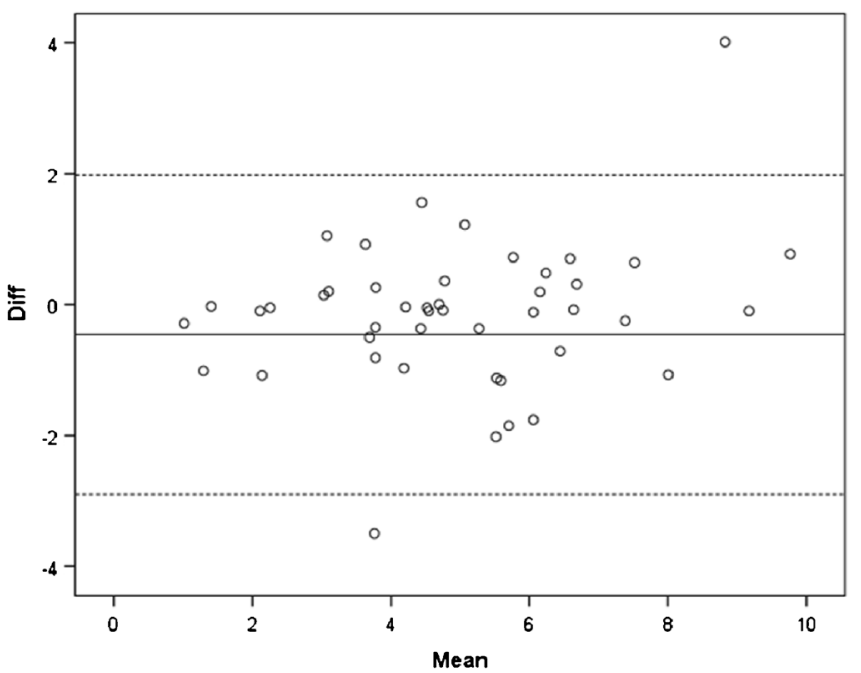

T2 GTV

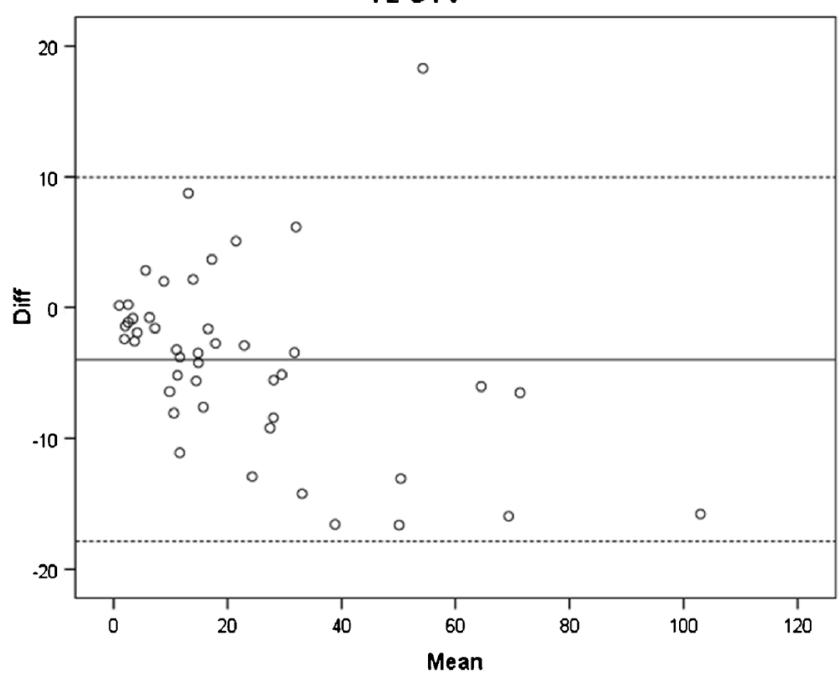

w, a relative difference of $17.43 \%$; similarly, Regini et al. measured smaller GTVs on DWI, with relative differences of $3.04 \%-7.62 \%$ not reaching statistical significance. Squamous cell carcinomas are typically associated with
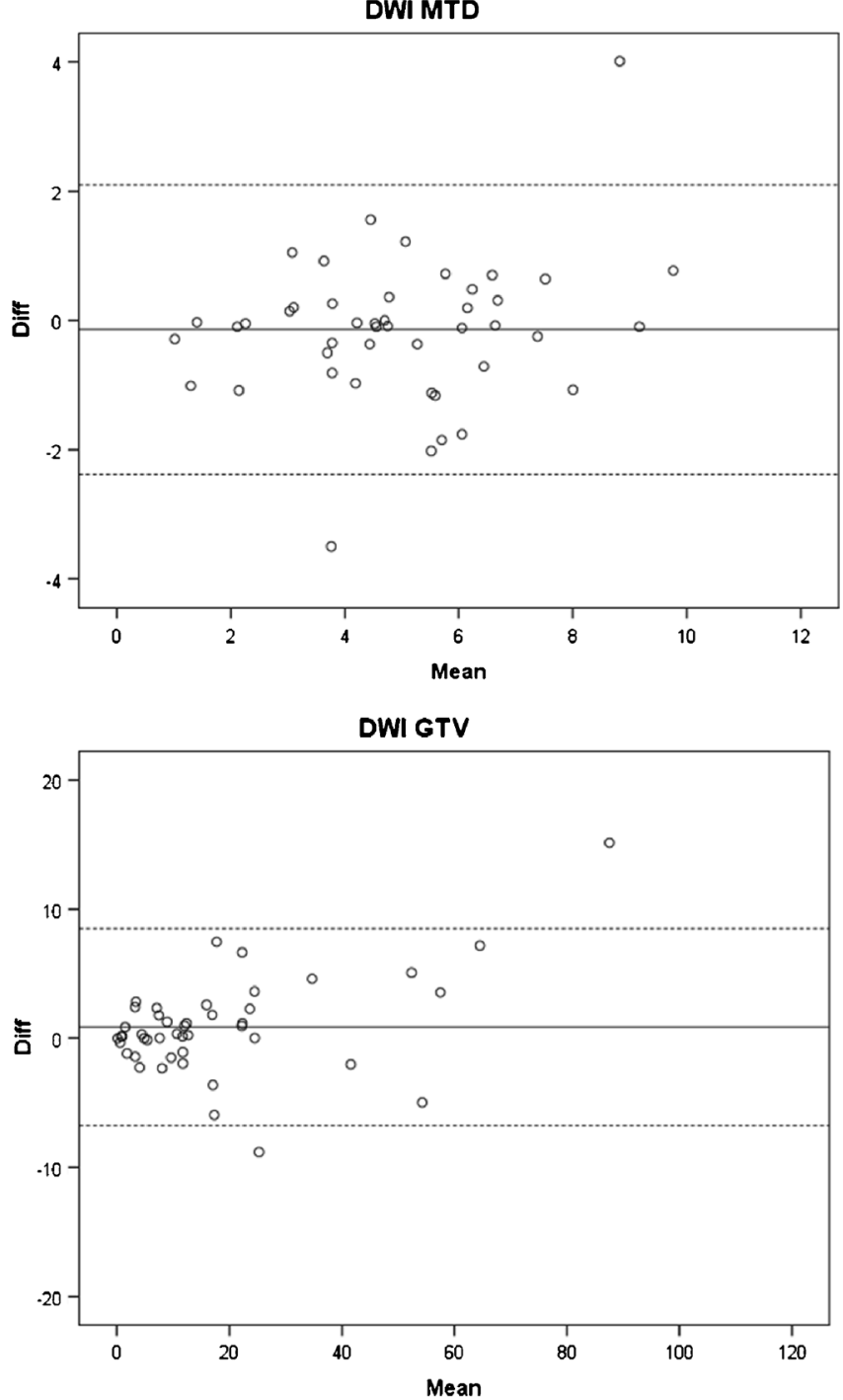

Fig. 4 Interobserver agreement. Bland-Altman plots for MTD and GTV on $\mathrm{T}_{2}$-w versus DWI sequences: relative interobserver differences (mean difference and $95 \%$ limits of agreement) are plotted against the mean value 
Table 3 Intraclass correlation coefficients (95\% confidence intervals)

\begin{tabular}{lll}
\hline & MTD & GTV \\
\hline $\mathrm{T}_{2}$-W & $0.899(0.803-0.946)$ & $0.968(0.915-0.985)$ \\
DWI $b 800$ & $0.925(0.863-0.959)$ & $0.990(0.918-0.994)$ \\
\hline
\end{tabular}

markedly impeded diffusion and display high signal intensities on DWI; adenocarcinomas, conversely, only appear moderately restricted because of their glandular structure and presence of mucin [22]. This pathological difference is likely to contribute to smaller DWI measurements in anal cancer compared to $\mathrm{T}_{2}-\mathrm{W}$ sequences.

Tumour greatest dimension is the only measurement determining $\mathrm{T}$ stage in SCCA according to AJCC TNM criteria [16]: based on our results, tumours bordering $2 \mathrm{~cm}$ and $5 \mathrm{~cm}$ in MTD (corresponding to T1/T2 and T2/T3 thresholds, respectively) are prone to categorisation variability, depending on both the reader and the sequence chosen for measurement: approximately a fourth of cases in our series were assigned a discordant $\mathrm{T}$ stage between $\mathrm{T}_{2}$-W and DWI sequences by both the inexperienced and experienced observer. With the wider implementation of personalised radiotherapy protocols, MTD and, consequently, T stage may also affect the GTV to clinical target volume (CTV) margin, the dose to the primary tumour and the use of simultaneous boost; the PLATO (Personalising Anal Cancer Radiotherapy Dose) protocol, for example, mandates an isocentric GTV-CTV margin of $10 \mathrm{~mm}$ for tumours up to $4 \mathrm{~cm}$ in MTD versus $15 \mathrm{~mm}$ for larger tumours [23]. To our knowledge, to date no other study has described the scale of this potential modality-, sequence- and observer-dependent variability and specific guidelines are still lacking on the matter.

Accurate tumour delineation is critical to radiotherapy planning. With the implementation of intensity modulated radiotherapy (IMRT) in the treatment of SCCA, it has become possible to escalate the dose to the target volume whilst maintaining the same or reducing dose to the surrounding normal tissues, resulting in steep dose gradients. To ensure appropriate dose delivery, both tumour and normal tissues must be delineated in $3 \mathrm{D}$ with high precision in reference to advanced diagnostic imaging techniques, including functional imaging [24]. The importance of access to high-quality diagnostic imaging has been illustrated by the US-based RTOG 0529 phase II trial evaluating dose-painting IMRT in SCCA, in which the gross tumour was inaccurately delineated in $21 \%$ of cases [25].

MRI is recognised in Europe as the modality of choice for locoregional staging of SCCA because of its high soft tissue contrast and its ability to depict local tumour infiltration; most clinical oncologists will refer to diagnostic MRI images at the time of planning: these can be co-registered with planning CT images used for dose calculation. The limiting factor in this setting may be the lack of experience in MRI interpretation; $\mathrm{T}_{2}$-W sequences represent the bedrock of pelvic MRI for detailed anatomical interpretation but require an advanced level of knowledge of the relevant cross-sectional anatomy. Signal intensities of tumour, muscle, fat and bowel contents are often very similar and can be challenging to tell apart with confidence. We believe our results partly reflect the challenges of distinguishing tumour from normal tissue in the anorectum on anatomical T2-w sequences alone. Fourteen and nine cases were assigned a low confidence score (1 to 3 ) by the inexperienced and experienced observer, respectively; these corresponded to either small (T1/T2) tumours with irregular margins and an infiltrative behaviour through the anal sphincter complex or anorectal junctional tumours surrounded by mucosal oedema and/or luminal fluid (Fig. 1).

In this context, the typically bright appearance of SCCA against a dark background on high b-value DWI facilitates
T2-w

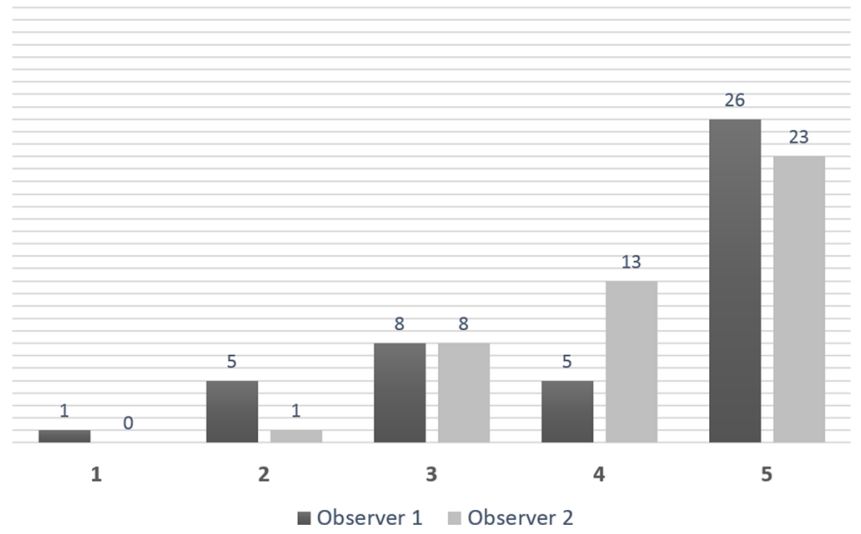

DWI

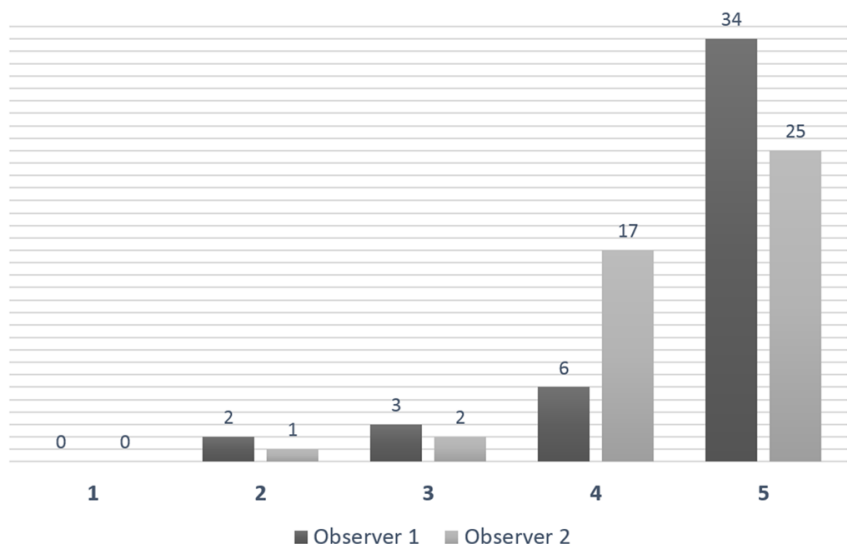

Fig. 5 Confidence scores. Both the inexperienced (Observer 1) and the experienced reader (Observer 2) outlined tumours confidently (scores of 4 to 5 ) more frequently on DWI than on $\mathrm{T}_{2}$-w. The confidence gain with DWI is greater for the inexperienced observer 
tumour delineation based on our study results. DWI certainly improved the confidence of both the inexperienced and experienced observer in outlining tumours in this study.

A drawback of the most commonly used single-shot echoplanar-imaging (EPI)-based DWI sequence is that it is prone to artefacts and susceptibility-related geometrical distortions, potentially detrimental in the setting of radiotherapy planning. These issues are being addressed through the development of distortion-correction strategies [26] and the optimisation of turbo spin echo (TSE)-based sequences [27]. In our high- $b$ value DWI series, the most common cause for measurement discrepancies between observers was the inclusion by the inexperienced observer of susceptibility artefacts at the anal verge (tissue-air interface), emphasising the importance of taking the learning curve into account when approaching DWI.

Regarding the potential implications of underestimating vs. overestimating tumour length/volume, it is worth stressing that the current research focus in patients with early disease is radiotherapy dose de-escalation, given the low rates of locoregional failure and significant toxicity at current dose regimens $[3,23]$. Conversely, patients with locally advanced disease, $30 \%$ of whom experience locoregional failure, may benefit from higher radiotherapy doses or sequential boosts by means of IMRT $[28,29]$. Applying these considerations to our study series and assuming experienced measurements as 'accurate', six cases would have been overstaged as T3 (advanced) disease by the inexperienced observer based on T2 sequences alone; none understaged; only 2 based on DWI (Fig. 3). Complementing T2 sequences with DWI, therefore, would seem more likely to save patients from radiotherapy toxicity than compromise their outcome by size underestimation.

This study has a number of limitations: its retrospective nature meant that minor variations in the imaging acquisition across different 1.5-T scanners could not be avoided; the sequences used for measurements and DWI $b$-values were nevertheless consistent. We did not evaluate spatial concordance and volume overlap between $T_{2}-\mathrm{W}$ and DWI, as performed by Burbach et al. for rectal cancer [30], though it would be interesting to assess the entity of geometrical distortions in anal cancer using conventional EPI-based DWI sequences. As DWI was acquired as a 2D axial sequence with a $1.5-\mathrm{mm}$ slice gap, sagittal reformats yielded slightly blurred images with a potential impact on MTD measurements: it is reassuring nevertheless that the trend for smaller measurements on DWI was maintained.

In summary, this study has shown that anal cancer MTD and GTV measurements are consistently and significantly lower on DWI than on T2-w sequences, with consequent intersequence $\mathrm{T}$ staging discordances and potential implications for radiotherapy target volume delineation. This highlights the need for more specific guidelines on the subjects.
Based on these findings and our clinical experience we would recommend the inclusion of DWI in anal cancer staging/ radiotherapy planning MRI protocols and its use alongside anatomical sequences. DWI measurements resulted in higher agreement between observers with differing levels of experience. DWI offered greater tumour delineation confidence over $\mathrm{T}_{2}$-W sequences to the inexperienced observer and even to the experienced in the case of small tumours infiltrating the anal sphincter complex or at the anorectal junction.

Funding The authors acknowledge financial support from the National Institute for Health Research via the Health Technology Assessment Programme; from the Department of Health via the National Institute for Health Research Comprehensive Biomedical Research Centre award to Guy's and St Thomas' NHS Foundation Trust, in partnership with King's College London and King's College Hospital NHS Foundation Trust; and from the King's College London/University College London Comprehensive Cancer Imaging Centre funded by Cancer Research UK and Engineering and Physical Sciences Research Council, in association with the Medical Research Council and Department of Health.

\section{Compliance with ethical standards}

Guarantor The scientific guarantor of this publication is Professor Vicky Goh.

Conflict of interest The authors of this manuscript declare no relationships with any companies, whose products or services may be related to the subject matter of the article.

Statistics and biometry No complex statistical methods were necessary for this paper.

Informed consent Written informed consent was waived by the Institutional Review Board.

Ethical approval Institutional Review Board approval was obtained.

\author{
Methodology \\ - retrospective \\ - observational \\ - performed at one institution
}

Open Access This article is distributed under the terms of the Creative Commons Attribution 4.0 International License (http:// creativecommons.org/licenses/by/4.0/), which permits unrestricted use, distribution, and reproduction in any medium, provided you give appropriate credit to the original author(s) and the source, provide a link to the Creative Commons license, and indicate if changes were made.

\section{References}

1. UK CR. Anal Cancer Statistics. http://www.cancerresearchuk.org/ health-professional/cancer-statistics/statistics-by-cancer-type/analcancer [07 June 2016]

2. National Cancer Institute. Surveillance E, and End Results Program. SEER Stat Fact Sheets: Anal Cancer. http://seer.cancer. gov/statfacts/html/anus.html [07 June 2016.] 
3. Glynne-Jones R, Nilsson PJ, Aschele C et al (2014) Anal cancer: ESMO-ESSO-ESTRO clinical practice guidelines for diagnosis, treatment and follow-up. Radiother Oncol 111:330-339

4. van der Heide UA, Houweling AC, Groenendaal G, Beets-Tan RG, Lambin P (2012) Functional MRI for radiotherapy dose painting. Magn Reson Imaging 30:1216-1223

5. Roach SC, Hulse PA, Moulding FJ, Wilson R, Carrington BM (2005) Magnetic resonance imaging of anal cancer. Clin Radiol 60:1111-1119

6. Koh DM, Dzik-Jurasz A, O'Neill B, Tait D, Husband JE, Brown G (2008) Pelvic phased-array MR imaging of anal carcinoma before and after chemoradiation. Br J Radiol 81:91-98

7. Goh V, Gollub FK, Liaw J et al (2010) Magnetic resonance imaging assessment of squamous cell carcinoma of the anal canal before and after chemoradiation: can MRI predict for eventual clinical outcome? Int J Radiat Oncol Biol Phys 78:715-721

8. Parikh J, Shaw A, Grant LA et al (2011) Anal carcinomas: the role of endoanal ultrasound and magnetic resonance imaging in staging, response evaluation and follow-up. Eur Radiol 21:776-785

9. Wagner M, Doblas S, Daire JL et al (2012) Diffusion-weighted MR imaging for the regional characterization of liver tumors. Radiology 264:464-472

10. Wagner M, Ronot M, Doblas S et al (2016) Assessment of the residual tumour of colorectal liver metastases after chemotherapy: diffusion-weighted MR magnetic resonance imaging in the peripheral and entire tumour. Eur Radiol 26:206-215

11. Bharwani N, Miquel ME, Powles T et al (2014) Diffusion-weighted and multiphase contrast-enhanced MRI as surrogate markers of response to neoadjuvant sunitinib in metastatic renal cell carcinoma. Br J Cancer 110:616-624

12. Sala E, Rockall AG, Freeman SJ, Mitchell DG, Reinhold C (2013) The added role of MR imaging in treatment stratification of patients with gynecologic malignancies: what the radiologist needs to know. Radiology 266:717-740

13. Jie C, Rongbo L, Ping T (2014) The value of diffusion-weighted imaging in the detection of prostate cancer: a meta-analysis. Eur Radiol 24:1929-1941

14. Lambregts DM, Lahaye MJ, Heijnen LA et al (2016) MRI and diffusion-weighted MRI to diagnose a local tumour regrowth during long-term follow-up of rectal cancer patients treated with organ preservation after chemoradiotherapy. Eur Radiol 26:2118-2125

15. Gourtsoyianni S, Goh V (2014) MRI of anal cancer: assessing response to definitive chemoradiotherapy. Abdom Imaging 39:2-17

16. Edge S, Byrd D, Compton C, Fritz A, Greene F, Trotti A (2010) AJCC cancer staging manual. 7th ed: Springer

17. Burnet NG, Thomas SJ, Burton KE, Jefferies SJ (2004) Defining the tumour and target volumes for radiotherapy. Cancer Imaging 4: 153-161

18. Bland JM, Altman DG (1999) Measuring agreement in method comparison studies. Stat Methods Med Res 8:135-160
19. Curvo-Semedo L, Lambregts DM, Maas M et al (2011) Rectal cancer: assessment of complete response to preoperative combined radiation therapy with chemotherapy-conventional MR volumetry versus diffusion-weighted MR imaging. Radiology 260:734-743

20. Regini F, Gourtsoyianni S, Cardoso De Melo R et al (2014) Rectal tumour volume (GTV) delineation using T2-weighted and diffusion-weighted MRI: Implications for radiotherapy planning. Eur J Radiol 83:768-772

21. Burbach JP, Kleijnen JP, Reerink O et al (2016) Inter-observer agreement of MRI-based tumor delineation for preoperative radiotherapy boost in locally advanced rectal cancer. Radiother Oncol 118:399-407

22. Lin M, Yu X, Chen Y, Ouyang H, Wu B, Zheng D, Zhou C (2017) Contribution of mono-exponential, bi-exponential and stretched exponential model-based diffusion-weighted MR imaging in the diagnosis and differentiation of uterine cervical carcinoma. Eur Radiol 27:2400-2410

23. UK Clinical Trials Gateway. PLATO - personalising anal cancer radiotherapy dose. Available via https://ukctg.nihr.ac.uk/trials/ trial-details/trial-details?trialNumber $=$ ISRCTN 88455282. Accessed on 16 Aug 2017

24. (2010) The international commission on radiation units and measurements. J ICRU 10(2):5-6

25. Kachnic LA, Winter K, Myerson RJ et al (2013) RTOG 0529: a phase 2 evaluation of dose-painted intensity modulated radiation therapy in combination with 5-fluorouracil and mitomycin-C for the reduction of acute morbidity in carcinoma of the anal canal. Int J Radiat Oncol Biol Phys 86:27-33

26. deSouza NM, Orton M, Downey K et al (2016) Distortion correction of echo-planar diffusion-weighted images of uterine cervix. J Magn Reson Imaging 43:1218-1223

27. De Foer B, Vercruysse JP, Pilet B et al (2006) Single-shot, turbo spin-echo, diffusion-weighted imaging versus spin-echo-planar, diffusion-weighted imaging in the detection of acquired middle ear cholesteatoma. AJNR Am J Neuroradiol 27:1480-1482

28. Geltzeiler CB, Nabavizadeh N, Kim J et al (2014) Chemoradiotherapy with a radiation boost for anal cancer decreases the risk for salvage abdominoperineal resection: analysis from the national cancer data base. Ann Surg Oncol 21:3616-3620

29. Deenen MJ, Dewit L, Boot H, Beijnen JH, Schellens JH, Cats A (2013) Simultaneous integrated boost-intensity modulated radiation therapy with concomitant capecitabine and mitomycin $\mathrm{C}$ for locally advanced anal carcinoma: a phase 1 study. Int J Radiat Oncol Biol Phys 85:e201-e207

30. Fleckenstein J, Jelden M, Kremp S et al (2016) The impact of diffusion-weighted MRI on the definition of gross tumor volume in radiotherapy of non-small-cell lung cancer. PLoS One 11: e0162816 